\title{
Detective Method for Water Pollution Based on Millimeter Wave Radiant Characteristics
}

\author{
Beibei Li, Guangfeng Zhang, Guowei Lou, Luyan Zhou, Jing Liu \\ School of Electronic and Optical Engineering, NanJing University of Science and Technology, Nanjing, China \\ Email: libeibei1990@163.com
}

Received 18 December 2015; accepted 26 February 2016; published 2 March 2016

\begin{abstract}
Aim at monitoring water pollution, especially the aquatic vegetation, the multilayer dielectric model based on incoherent method is established to analysis the brightness temperature of aquatic vegetation. A $3 \mathrm{~mm}$ radiometer is used to measure the radiant characteristics of water pollution. Compared to 3 layer dielectric model, the simulation result of multilayer dielectric model is in better accordance with the experimental data, which shows that the multilayer dielectric model can model aquatic vegetation's radiant characteristics more precisely. This result shows that water has millimeter wave radiant characteristics of low brightness temperature, cold target compared to aquatic vegetation. Based on the study of water's brightness temperatures and aquatic vegetation's radiant characteristics, the radiant characteristics can be used to monitor aquatic vegetation.
\end{abstract}

\section{Keywords}

Multilayer Dielectric Model, Millimeter Wave, Radiant Characteristic

\section{Introduction}

Along with the economic development, water pollution is becoming one of the most serious environmental problems. At present, most of the detection methods of water pollution are staying in visible light and infrared model, these ways are usually affected by environment or weather. Compared with the light and infrared model, the millimeter wave has the advantages of all-weather, high detection accuracy and good invisibility [1]. These characteristics make it widely used in agriculture, geology, environment, surveying and mapping, military and other fields.

Aiming at the water pollution, Qulin Tan use multi-source remotely sensed data (Landsat TM/ETM, Radarsat SAR and Terra Modis etc.) related documents and geographical data, statistical data of water level and area through many years to identify and monitor the environment of PoYang Lake [2]. Ganlin Wang analysis the combine of Modis and SAR (synthetic aperture radar) to monitor algal blooms in Taihu Lake, which meet acquirement of accuracy and timeliness in remote sensing field.

There are many types of water pollution, such as white pollution, vegetation pollution, factory pollution, etc, this paper will focus on aquatic vegetation pollution, cause the aquatic vegetation is not only a kind of pollutants, 
can also provide a way to control water pollution sometimes.

For water pollution such as oil, aquatic vegetation, the most commonly used to model is 3 layers model [3]. Considering there are gaps between aquatic vegetation, 3 layers model is not suitable to be used for aquatic vegetation. This paper applies the multilayer dielectric model to analysis the radiation characteristics of aquatic vegetation, the multilayer dielectric model is based on incoherent method. Compared with common water pollutants, water has low bright temperature, cold target radiation characteristics, water pollutions and water can be distinguished through these features.

\section{Multilayer Dielectric Model and Simulation}

Pollution of aquatic vegetation has characteristics of stacking with each other, air layer exists between aquatic vegetation, based on this, in order to study the millimeter wave radiation characteristics of aquatic vegetation pollutants, a multilayer microwave transmission model for aquatic should be established.

\subsection{Multilayer Dielectric Model}

Generally, the classical model for pollutions is 3 layers, as for aquatic vegetation, because the aquatic vegetation had air gap, the multi-layer model can be applied to analyze the bright temperature, multilayer dielectric model is discussed in [4], Wang extended Ulaby incoherent method to $\mathrm{N}(\mathrm{N}>3)$ layers [5]. The emission of each layer can be calculated by Rayleigh-Jeans approximation, the model is shown in Figure 1.

$T_{B}$ is the total radiation temperature of the aquatic vegetation pollutant which is observed by the radiometer, its value is equivalent to the radiation temperature of the first layer of the aquatic vegetation and the temperature of the radiation of all the medium layers:

$$
T_{B}\left(\theta_{1}, p\right)=T_{B 1}\left(\theta_{1}, p\right)+T_{L 1}\left(\theta_{1}, p\right)
$$

where $\theta_{1}$ is the electromagnetic wave incident angle of the first layer of aquatic vegetation, $p$ is the polarization of electromagnetic wave, $T_{L 1}$ is the second layer of the medium layer below the radiation temperature of all the media. $T_{B 1}$ is the first layer of aquatic vegetation radiation brightness temperature,

$$
T_{B 1}=T_{2 U}+T_{2 D}=\frac{1-\Gamma_{1}}{1-\Gamma_{1} \Gamma_{2} / L_{2}^{2}}\left(1+\frac{\Gamma_{2}}{L_{2}}\right) T_{s 2}
$$
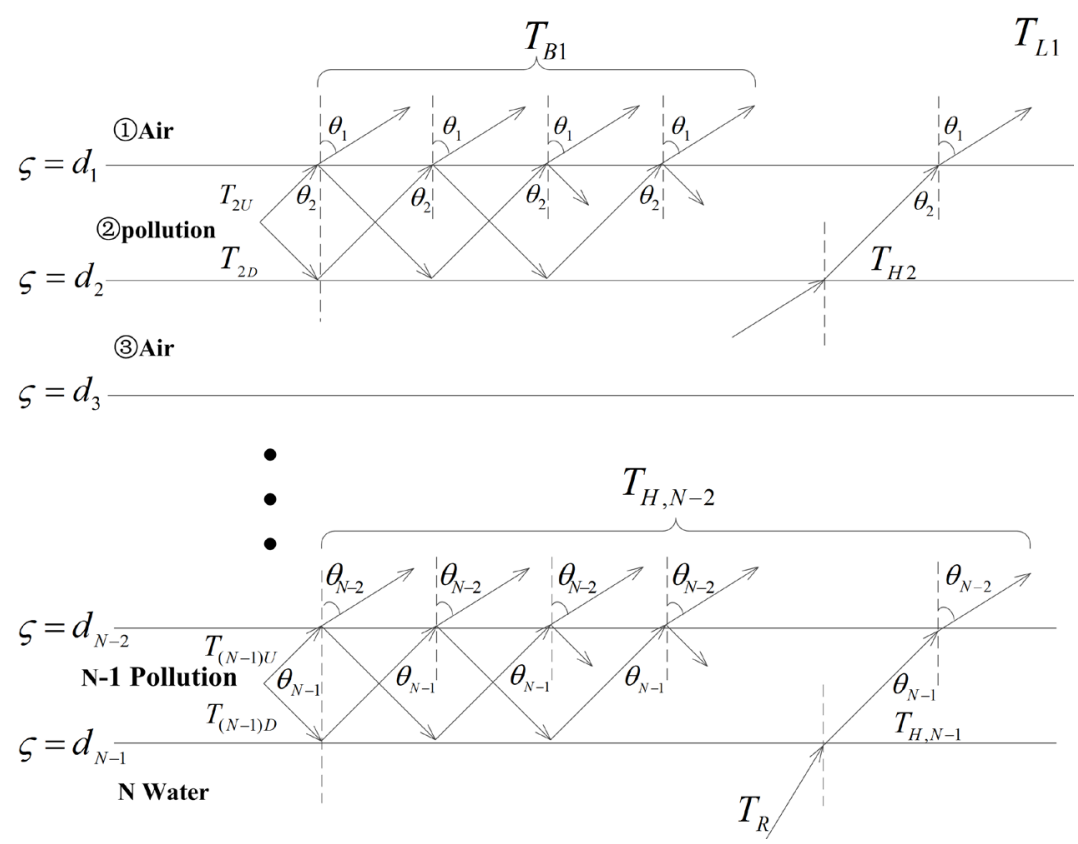

Figure 1. Multilayer dielectric model based on incoherent method. 
where $T_{2 U}$ and $T_{2 D}$ are electromagnetic waves radiated from the vegetation, $\Gamma_{1}$ is the reflectivity of first layer and the second layer, $\Gamma_{2}$ is the reflectivity of second layer and third layer medium, $L_{2}$ is defined as the second layer medium power loss factor, $T_{s 2}$ is the second layer medium total spontaneous radiation.

$T_{H, \mathrm{i}-1}$ is the $r$ radiation temperature of layer $i$ and all of the following dielectric layer radiation temperature reach the boundary

$$
T_{H, i-1}=\frac{1-\Gamma_{i-1}}{1-\Gamma_{i-1} \Gamma_{i} / L_{i}^{2}}\left[\left(1+\frac{\Gamma_{i}}{L_{i}}\right) T_{s i}+\frac{1}{L_{i}} T_{H, \mathrm{i}}\right]
$$

where $T_{s i}$ is the total spontaneous radiation of layer $i$.

$$
T_{s i}=\int_{d_{i-1}}^{d_{i}} \sec \theta_{i} k_{a i} T_{i} e^{-\sec \theta_{i} k_{a i}\left(\xi-d_{i-1}\right)} d \zeta=\left(1-a_{i}\right)\left(1-\frac{1}{L_{i}}\right) T_{i}
$$

where $a_{i}=k_{s i} / k_{e i}$, is single-scattering albedo of the layer $i, k_{e i}$ is its extinction coefficient, $k_{s i}$ is scattering coefficient.

Assumed the water is infinite, $T_{H, N-1}$ is radiation temperature of the water body, which is at the upper part of the interface between the layer $N-1$ and the layer $N$,

$$
T_{H, N-1}=\left(1-\Gamma_{N-1}\right) T_{N}
$$

\subsection{Simulation}

According to the established aquatic vegetation multilayer dielectric model, taking the angle of incidence of $45^{\circ}$, because each overlap a leaf will also add a layer of air gap layer, so the $\mathrm{N}$ layers of simulation were taken for 3 , 5, 7. Figure 2 shows the simulation of emissivity and reflectivity of aquatic vegetation change along with the thickness variation of aquatic vegetation,

From the simulation, we can know that the emissivity increases with the increase of thickness. The reflectivity decreases with the increase of the thickness, as the thickness increases, the emissivity and reflectivity gradually become stable.

\section{Experiments and Analysis}

In order to verify the correctness of the multilayer dielectric model of the aquatic vegetation, the radiation characteristics of aquatic vegetation and water surface were measured by using the $3 \mathrm{~mm}$ radiometer. The experiment was carried out in Taihu Lake.



Figure 2. Emissivity and reflectivity based on multilayer dielectric model. 


\subsection{Experiments}

Figure 3 shows the experiment environment, experiment conditions: the temperature is $34^{\circ} \mathrm{C}$, the humidity is $44 \%$, the incidence angle is $50^{\circ}, 55^{\circ}, 45^{\circ}, 60^{\circ}$ and $65^{\circ}$, respectively. When incidence angle beyond the range, the data would be influenced by the antenna and surrounding environment, the data have no practical significance, Table 1 shows the measurement results.

\subsection{Analysis}

According to the multilayer dielectric model established above, when $\mathrm{N}$ is 3, 5, 7, respectively ,the simulation data of the relationship between the brightness temperature of the aquatic vegetation and the incident angle is shown in Figure 4, for the convenience of comparative analysis, the experimental data is also given in Figure 4. The experimental results are found to be in good agreement with the simulated results.

The results are evaluated in the independent checking samples. Table 2 gives the RMSE (root mean square error) of the simulation data. When $\mathrm{N}$ is equal to 7, the RMSE is the smallest, but at the same time, the computational complexity is higher. Compared to the 3 layers model, when $\mathrm{N}$ is equal to 5 , the accuracy has been greatly improved, in the case of requirements of accuracy is not strict, choose 5 layer model to simulate is more suitable.

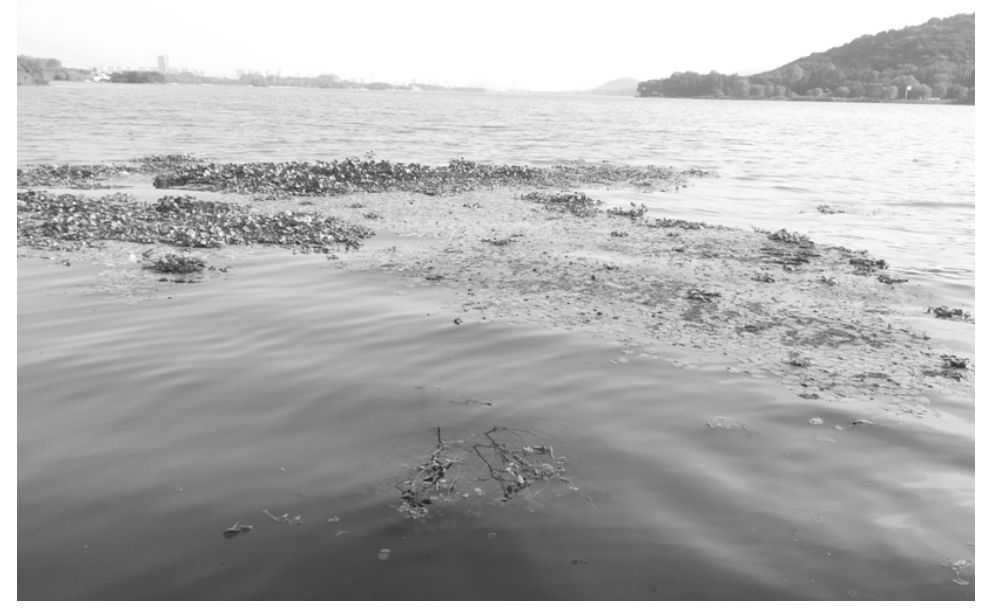

Figure 3. Experiment environment.

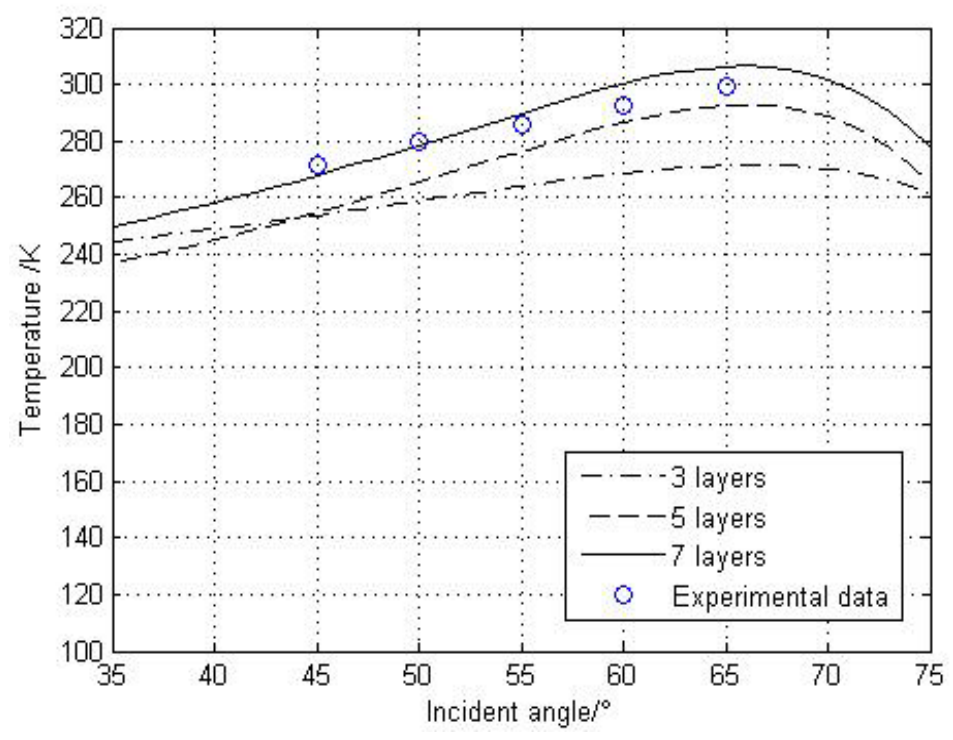

Figure 4. Experimental data and simulation of multilayer dielectric model. 
Table 1. Radiation temperature of 3 mm radiometer.

\begin{tabular}{rccccc}
\hline Incidence Angle $\left(^{\circ}\right)$ & 45 & 50 & 55 & 60 & 65 \\
Radiation temperature $(\mathrm{K})$ & 271.9 & 279.9 & 286.2 & 292.8 & 299.7 \\
\hline
\end{tabular}

Table 2. Root mean square error of experimental data.

\begin{tabular}{cccc}
\hline Layers & 3 layers & 5 layers & 7 layers \\
\hline RMSE $(\mathrm{K})$ & 22.3 & 11.8 & 5.1 \\
\hline
\end{tabular}

\section{Conclusions}

In this paper, a microwave multilayer dielectric model is analyzed, and is used to study the radiation characteristics of aquatic vegetation. The model divides aquatic vegetation, air and water into different layers with different dielectric constants and physical temperatures. The integration is carried out by summing up each layer's emissions and attenuations, while considering multi-reflection of the up and down boundaries. Based on the experimental data, the multilayer dielectric model aquatic vegetation's radiant characteristics more precisely compared with 3 layers model.

On the basis of these results, we conclude that water has low bright temperature, cold target radiation characteristics, compared with common water pollutants. For practical applications, water pollutions and water can be distinguished through these features.

\section{Acknowledgements}

This work has been partially supported by the Natural Science Foundation of China (Project No. 61371038), Natural Science Foundation of Jiangsu (Project No. BK2010490).

\section{References}

[1] Dong, N.H. and Wu, G.H. (1996) The Study of Millimeter Wave Remote Sensing System in the Application of Reentering Environment. Proceedings of 4th International Conference on Millimeter Wave and Far Infrared Science and Technology, Beijing, 12-15 August 1996, 121-124. http://dx.doi.org/10.1109/ICMWFT.1996.574789

[2] Tan, Q., Shao, Y., Yang, S. and Wei, Q. (2003) Landsat TM Optimal Bands Selection for Freshwater Lake International Importance Wetland Interpretation and Monitoring. Proceedings of IEEE International Geoscience and Remote Sensing Symposium, 5, 3299-3301. http://dx.doi.org/10.1109/IGARSS.2003.1294762

[3] Wang, Z.Z., et al. (2008) Microwave Transfer Models and Brightness Temperature Simulations of MWS for Remote Sensing Lunar Surface on CE-1 Satellite. International Conference on IEEE Microwave and Millimeter Wave Technology, Nanjing, 21-24 April 2008, 1683-1686. http://dx.doi.org/10.1109/ICMMT.2008.4540793

[4] Wang, Z., Li, Y., Jiang, J., Dong, X., Zhang, X., Zhang, D., et al. (2008) A Microwave Radiative Transfer Model Applied to Lunar Soil Remote Sensing. Automation Congress, WAC World, 1-4.

[5] Ulaby, F.T. and El-Rayes, M. (1987) Microwave Dielectric Spectrum of Vegetation-Part II: Dual-Dispersion Model. IEEE Transactions on Geoscience \& Remote Sensing, GE-25, 550-557. http://dx.doi.org/10.1109/TGRS.1987.289833 